(C2008 IEEE. Personal use of this material is permitted. However, permission to reprint/republish this material for advertising or promotional purposes or for creating new collective works for resale or redistribution to servers or lists, or to reuse any copyrighted component of this work in other works must be obtained from the IEEE 


\title{
Enhanced Real-Time ECG Coder for Packetized Telecardiology Applications
}

\author{
Álvaro Alesanco, Salvador Olmos, Member, IEEE, Robert S. H. Istepanian, Senior Member, IEEE, and José García
}

\begin{abstract}
A new real-time compression method for electrocardiogram (ECG) signals has been developed based on the wavelet transform approach. The method is specifically adaptable for packetized telecardiology applications. The signal is segmented into beats and a beat template is subtracted from them, producing a residual signal. Beat templates and residual signals are coded with a wavelet expansion. Compression is achieved by selecting a subset of wavelet coefficients. The number of selected coefficients depends on a threshold which has different definitions depending on the operational mode of the coder. Compression performance has been tested using a subset of ECG records from MIT-BIH Arrhythmia database. This method has been designed for realtime packetized telecardiology scenarios both in wired and wireless environments.
\end{abstract}

Index Terms-Compression, electrocardiogram (ECG) coder, electrocardiogram (ECG) transmission, telecardiology.

\section{INTRODUCTION}

$\mathbf{T}$ ELECARDIOLOGY is considered one of the most mature and successful e-Health applications. This is demonstrated by the large amount of telecardiology projects that have been carried out in the last years [1]-[4]. There are two different ways in which electrocardiogram (ECG) data can be transmitted in a telecardiology environment: real-time and store-and-forward (prerecorded) transmission. Store-and-forward ECG transmission has been widely used in earlier telecardiology systems where real-time ECG interpretation and/or supervision was not crucial. An example of store-and-forward ECG transmission would be that of a General Practitioner (GP) recording an ECG at patient's home transmitted to a cardiologist for analysis and interpretation. Although store-and-forward ECG transmission has been extensively used due to its simplicity and efficiency, there are more critical scenarios where real-time ECG transmission is required, if not essential. Emergency situations reveal the critical application of such real-time wireless telecardiology systems. Several studies have shown that 12-lead ECG performed within an ambulance increases the critical available time to administer thrombolytic therapy effectively, stopping

Manuscript received October 21, 2004; revised March 5, 2005.

This work was supported by Project TSI2004-04940-C02-01 from the Comisión Interministerial de Ciencia y Tecnología (CICYT) and Fondos Europeos de Desarrollo Regional (FEDER), by Project FIS G03/117 from the Fondo de Investigación Sanitaria (FIS), and by Telefónica Móviles of Spain.

Á. Alesanco, S. Olmos, and J. García are with the Communications Technology Group, Aragón Institute of Engineering Research, University of Zaragoza, 50018 Zaragoza, Spain (e-mail: alesanco@unizar.es).

R. S. H. Istepanian is with the Mobile Information and Network Technologies Centre, School of Computing and Information Systems, Kingston University, London, KT1 2EE, U.K.

Digital Object Identifier 10.1109/TITB.2005.856853 a heart attack in progress and preserving heart muscle function [5]. Although store-and-forward ECG transmission is also possible from the ambulance to the hospital, real-time ECG monitoring by a cardiologist in a hospital would be more convenient because it allows a reduction in the time needed for patient's evaluation once it arrives to the hospital. These effects are specially crucial in cases where the transportation time is long [6], [7].

During the last years, the increasing popularity of Internet has made that the TCP/IP protocol stack has been implemented in the network and transport layers in almost every communication network [8]. Wireless networks which formerly were implemented as circuit-switching have migrated to packet-switching implementing the TCP/IP protocol stack [9]. This is especially the case of third generation (3G) wireless networks. However, the recent advances in $2.5 / 3 \mathrm{G}$ wireless communications are not paralleled in similar advances on using dedicated ECG packetization schemes for real-time telecardiology applications and no study yet has addressed the issues of packet transmission dedicated for telemedical systems.

The development of efficient real-time compression methods for ECG data transmission in limited bandwidth wireless networks such as the General Packet Radio Service (GPRS) systems is crucial for a wider use of such wireless telemedical services in healthcare systems. It is well known that in order to use the available network bandwidth in an efficient way, a compression algorithm is required. ECG compression is a well-studied topic. In the last decade, many ECG coders have been presented [10]-[12]. Nevertheless, most of these studies direct their efforts in store-and-forward compression, leaving real-time operation as a possible future application without giving any specific results in this area. To the authors knowledge no study is reported yet on wavelet compression scheme suitable for packetized (wired or wireless) telecardiology applications. Although in [13] a real-time ECG transmission system is described, ECG data is transmitted without compression and with no detail about packetization issues.

In this paper, we present a new real-time ECG coding scheme compatible with packetized telecardiology applications. The method is based on the wavelet transform of aligned beats. Each beat is compressed to target a desired bit rate or a reconstruction error, depending on the operational mode. Finally, a packet is built to transport the coded beat to the receiver.

The paper is organized as follows. In Section II the compression method is presented. The simulation results and the discussion of the performance of the method in a packetized transmission environment are shown in Section III. Finally, conclusions are reported in Section IV. 


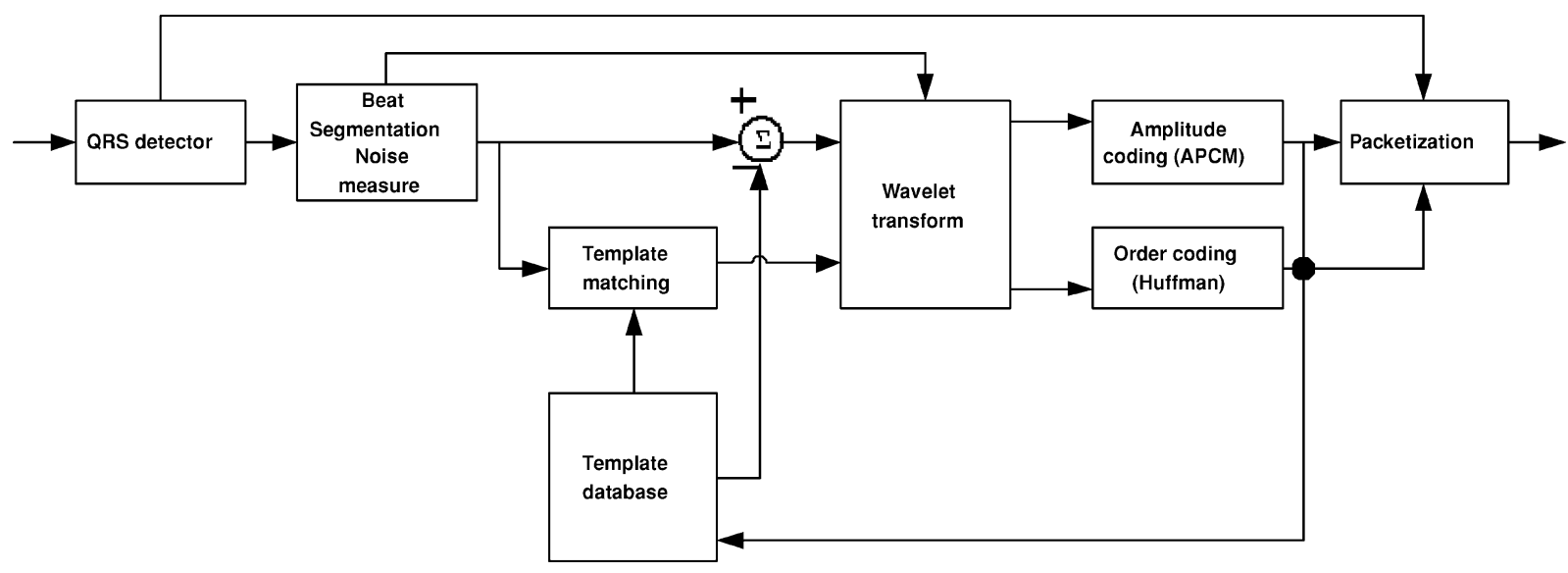

Fig. 1. Block diagram of the packetized ECG compression system.

\section{COMPRESSION METHOD}

The compression method is structured in four stages:

i) preprocessing: including baseline removal, QRS detection and noise measure;

ii) template subtraction;

iii) wavelet transform: coefficient selection and coding schemes;

iv) data packetization.

A general scheme of the compression method is shown in Fig. 1. The description of each block in the process is given as follows.

\section{A. Preprocessing: Baseline Removal, QRS Detection, and Noise Measure}

ECG signals usually contain very low frequency components originated by noncardiac sources such as respiration and relative movements of electrodes and skin. As real-time operation is required, a simple ECG baseline estimation method is used: a third-order Butterworth low-pass filter (cut-off frequency equal to $0.5 \mathrm{~Hz}$ ) used in forward/backward directions to avoid phase distortion. After this estimation, baseline is subtracted from the ECG signal.

For QRS detection a previously developed algorithm based on the wavelet transform is used [14]. The ECG is segmented into beats aligned from their QRS fiducial points. A beat is defined beginning at $250 \mathrm{~ms}$ prior to its QRS fiducial point. Every beat has a dyadic length by padding with the beat last sample amplitude. If the ECG signal has more than one lead, each lead is independently processed along the coding. Once a beat has been detected and segmented from the multilead ECG signal, it is represented as a multilead beat vector. If a QRS is not detected within an interval equal to the wavelet transform length (the dyadic length commented previously), the ECG is segmented at this time and the acquired signal is passed to the next stage.

Noise power in each beat is estimated as the power remaining after high-pass filtering (cutoff frequency equal to $20 \mathrm{~Hz}$ ) in the repolarization interval. This measure will be useful to set up the compression threshold.

\section{B. Template Subtraction}

A template (which is a multilead beat vector as well) is subtracted to the multilead beat vector, yielding a multilead residual signal. The method makes use of a dynamic template database which is created, modified and updated in real-time using the new acquired beats. The first template stored in the database is the first available multilead beat vector of the signal after being coded. Then, for each new acquired beat the normalized correlation coefficient [15] is calculated between the QRS complex interval (defined from $100 \mathrm{~ms}$ prior the QRS fiducial point to $100 \mathrm{~ms}$ after it) and every template in the template database. This coefficient is only computed on one lead because beat morphology changes usually appear in a congruent way in every lead. The template assigned to be subtracted to the multilead beat will be the one with the largest normalized correlation coefficient, unless this value is low (below 0.8); in that case, a new multilead beat template is created and added to the database with the current coded multilead beat vector (as it will be reconstructed in the receiver). In order to take into account beat morphology variations, multilead beat templates are updated after being used 10 times for beat subtraction. This number was heuristically determined (tests showed that an increase in the updating frequency did not lead to a relevant increase in the performance). The new template is calculated as the running average of the current coded multilead beat vector and the old template. It is worthy to note that the coded signal is used for template updating and thus no additional information is needed for the updating process in the receiver.

When a block coming from a nondetected QRS event has to be coded, a special template is used. This template is a constant zero value (noted as zero template). In this way, signals coming from disconnection events are coded as pure residuals since their associated template has a constant zero value. There is no need for a special treatment for these events: they simply use the zero template as associated template.

\section{Wavelet Transform (WT): Coefficient Selection and Coding Schemes}

The residual signals as well as beat templates are coded by means of a Coiflet wavelet expansion [16] [see an example 


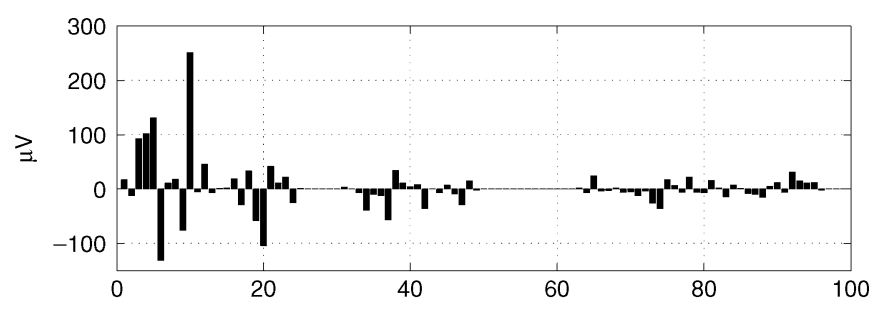

(a)

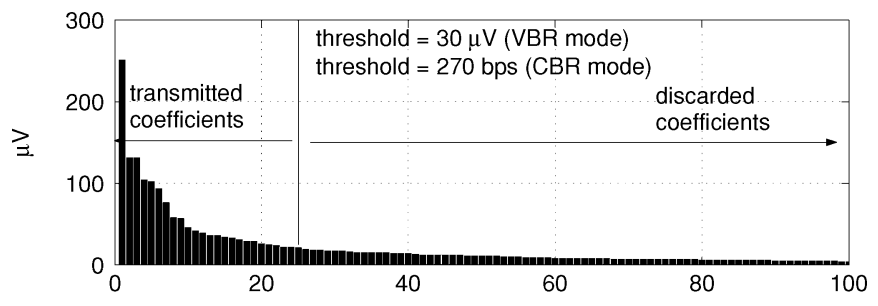

(b)

Fig. 2. Wavelet coefficients selection process for a residual signal. WT length $=512$ samples. (a) Wavelet coefficients. (b) Wavelet coefficients sorted by abs (amplitude).

in Fig. 2(a)]. Other wavelets were initially considered (such as biorthogonal 9/7 [12], [11]) but no superior performance was found with them. Each lead is processed independently, as commented previously. Compression is achieved by selecting a subset of wavelet coefficients and coding them in an efficient way. The coefficients are sorted by absolute amplitude order and the number of coefficients to be transmitted is selected according to a threshold defined depending on the operational mode [see Fig. 2(b)].

The number of selected (transmitted) coefficients is defined by a threshold. In this method two different operational modes are permitted: variable bit rate (VBR) and constant bit rate $(\mathrm{CBR})$. When the system operates in VBR mode, the threshold, measured in $\mu \mathrm{V}$ [see Fig. 2(b)], is defined to achieve a fixed Root Mean Square (RMS) reconstruction error by rejecting the smallest coefficients until their accumulated power reaches the threshold.

A variable number of coefficients are needed to achieve the fixed RMS error since the residual signal morphology varies beat-to-beat. In our implementation, the VBR mode presents two operational modalities depending on how the threshold is established. It can be setup to achieve a desired constant reconstruction error within a signal interval (VBR with constant threshold). The other option is to change beat-to-beat the threshold in order to remove white noise from it (VBR with variable threshold). It is wellknown that white noise presents a uniform distribution in any domain while ECG signal energy is concentrated in a small group of coefficients with low frequencies. Thus, the signal-to-noise ratio (SNR) can be improved by selecting the coefficients with largest amplitude from the lower scales of the wavelet transform. If the noise level increases, the number of WT coefficients should be decreased in order to avoid spending bit rate for noise coding. Then, in this mode, the number of WT coefficients is selected in order to assure that the power of the rejected coefficients equals the noise power estimated in the preprocessing stage. This is the optimum point for white noise removal as it is shown in Fig. 3.

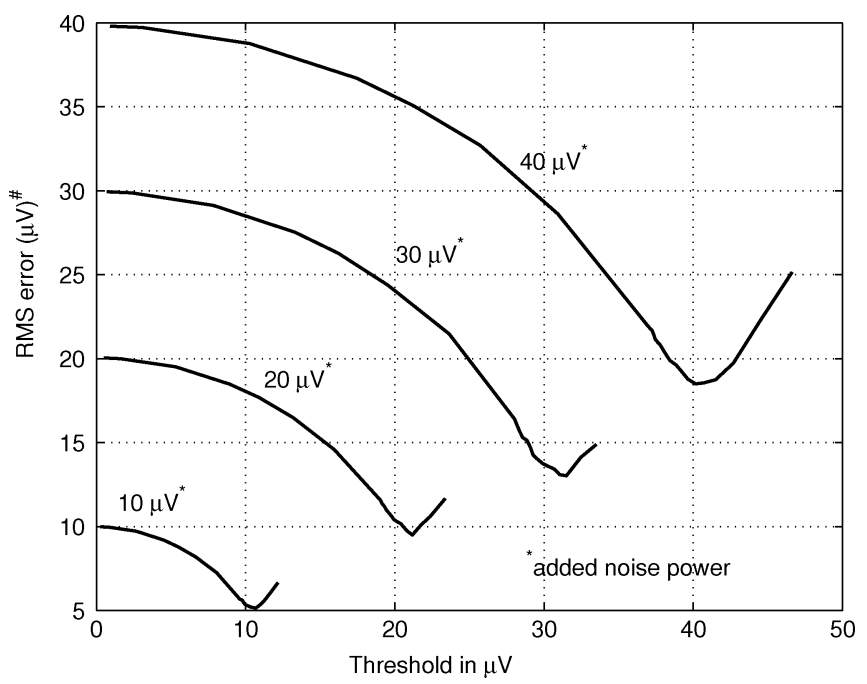

Fig. 3. Optimum coding point for VBR mode with variable threshold. \# RMS has been calculated between noiseless and reconstructed signals, compressed from the noisy signals.

To obtain the curves shown in Fig. 3, noisy ECG signals were simulated by adding zero-mean white noise with several noise levels $(10,20,30$, and $40 \mu \mathrm{V})$ to resting ECG records (noise level below $2 \mu \mathrm{V}$, so we call them noiseless signals). The sampling frequency was $256 \mathrm{~Hz}$ with a resolution of 16 bits per sample. The compression threshold has been ranged from $0 \mu \mathrm{V}$ (all the coefficients are transmitted) to $5 \mu \mathrm{V}$ above the added noise power. The optimum threshold for which the RMS is minimum is reached when the power of the discarded coefficients equals the power of the noise added. Therefore, there is no advantage on increasing the subset of signal coefficients transmitted beyond the value defined by the optimum threshold since if new coefficients are added, an increase in the reconstruction noise will be obtained. Note that RMSerror in Fig. 3 is calculated between the reconstructed signal (which has been compressed from the noisy signal) and the noiseless signal. Thus, when all the coefficients are used to code the noisy signal (threshold $=0 \mu V$ ), the reconstruction error is equal to the added noise (as it can be seen in Fig. 3) and not equal to $0 \mu \mathrm{V}$ as it would be obtained if the error is calculated between the noisy signal and the reconstructed signal. Since a subset of coefficients is removed, this process leads also to signal compression. The threshold is thus beat-to-beat automatically updated according to the measured noise level.

If the operation mode is CBR, the threshold, which is defined in bits per second (bps), is set up to achieve a constant bit rate [see Fig. 2(b)]. The number of bits used for coefficients coding $\left(b_{c}(i)\right)$ (without considering the header information) is calculated beat-to-beat in the following way:

$$
b_{c}(i)=\mathrm{bps}_{d} \cdot \operatorname{rr}(i)
$$

where $\operatorname{bps}_{d}$ is the desired transmission rate and $\operatorname{rr}(i)$ is the $\mathrm{RR}$ interval of the $i$ th beat in seconds. Once $b_{c}(i)$ is obtained, the number of coefficients transmitted will be that one which after coefficient coding fits the number of bits 
available [Fig. 2(b)]. After the thresholding process, coefficients are sorted by order to return to the original coefficient sequence.

The WT coefficients orders and amplitudes need to be coded for each signal (residuals and templates). Since the power spectral density of the ECG signal has a smooth low-pass shape, the energy distribution at each scale of the wavelet transform will be similar for different beats and it concentrates in a few number of coefficients which amplitude decreases sharply, as shown in Fig. 2(b). It also makes that the relevant WT coefficients on each ECG block appear very close in the order sequence within a wavelet scale [see Fig. 2(a)]. The use of the first difference in a vector containing the orders of the selected coefficients makes that the proximity within scales of the orders of the relevant coefficients will lead to low values, that can be much more efficiently coded. Thus, the first difference of the selected coefficients orders (denoted as jumps [17]) can be coded with a Huffman code in a highly efficient way.

The histogram of the jumps when the number of selected WT coefficients is equal to 20 is shown in Fig. 4(b). This figure has been obtained using a population of more than 50 ECG records from different databases. Small jump values have much higher probability than large values [Fig. 4(b)]. Fig. 4(a) shows the probability evolution of the small jumps when varying the number of selected WT coefficients. For a better understanding of Fig. 4 consider the following example. Let amp $=[123,52,-231,20,76,29,-78]$ be the amplitudes of a hypothetical subset of selected coefficients for one beat and ord $=[1,2,3,10,12,13,24]$ be their orders. Therefore, jumps will be jumps $=[1,1,7,2,1,11]$. The number of selected coefficients in this example is 7 . The histogram of the jumps values will be hist $=[3,1,0,0,0,0,1,0,0,0,1]$ and the translation of this histogram to probabilities will be prob $=[0.5,0.166,0,0,0,0,0.166,0,0,0,0.166]$. If we repeat this process for every beat in the ECG signal always selecting 7 coefficients for transmission and we average the results, we will obtain a similar figure to Fig. 4(b) but for 7 coefficients instead for 20. If we repeat again this process but now we vary the selected coefficients from 1 to 512 (the WT length) and we represent for each subset of selected coefficients the mean probability values obtained for jumps from 1 to 4, we will obtain the figure shown in Fig. 4(a). In this way, Fig. 4(b) is a transversal cut for 20 coefficients of Fig. 4(a).

Variablity of the histograms within the training records was very small. Although different number of selected coefficients would need a different PDF to optimally store the jumps, the histogram corresponding to 20 WT coefficients was chosen for the Huffman code [see Fig. 4(b)]. The mean number of bits to code an order using the Huffman code varies depending on the number of selected coefficients [as it is suggested by the evolution of jump probabilities in Fig. 4(a)]. For example, when 20 coefficients are selectd, an average of 3.15 bits are used instead of the 9 bits that would be necessary if a PCM coder would be used (WT length equal to 512).

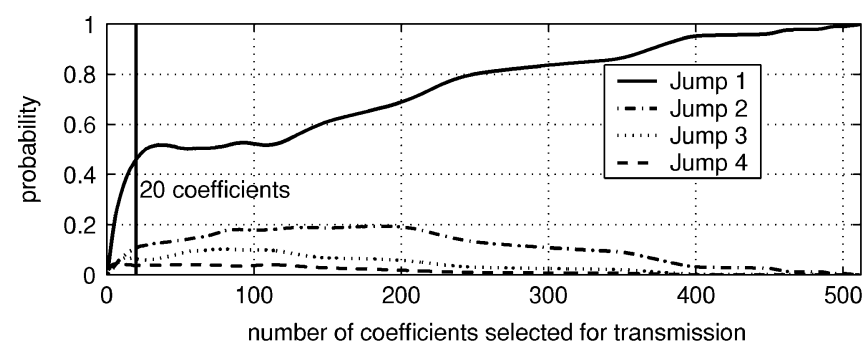

(a)

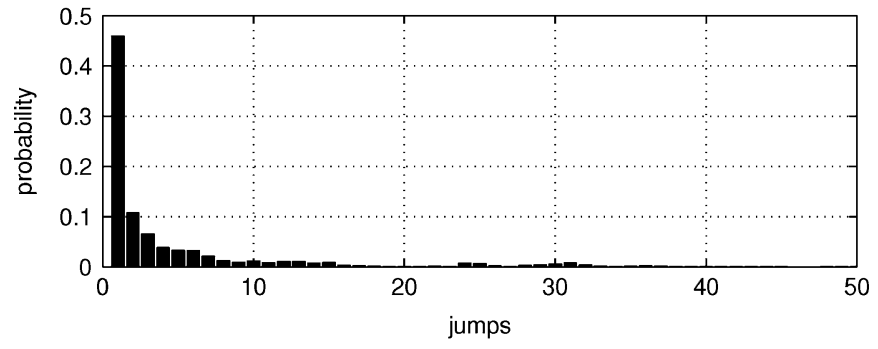

(b)

Fig. 4. Jumps histogram. (a) Evolution of the histogram from different jump values. (b) Histogram of the jumps for 20 selected coefficients.

\begin{tabular}{|c|c|}
\hline Template index (variable) & $\mathbf{N}$ of leads (3 bits) \\
\hline \multicolumn{2}{|c|}{$\mathbf{N}$ of coefficients for lead $j(j=1: n))$ (9 bits) } \\
\hline \multicolumn{2}{|c|}{$\mathbf{N}$ of bits to code coefficient amplitude in lead $j(j=1: n)$ (4 bits) } \\
\hline \multicolumn{2}{|c|}{ Coefficients amplitude for lead $j(j=1: n)$ (variable) } \\
\hline \multicolumn{2}{|c|}{ First coefficient order for lead $j(j=l: n)$ (9 bits) } \\
\hline \multicolumn{2}{|c|}{ Jumps for lead $j(j=l: n)$ (variable) } \\
\hline RR interval (9 bits) & \\
\hline
\end{tabular}

Fig. 5. PDU format used in the coding method. The number of bits used in each field is shown in brackets.

Finally, an adaptive pulse code modulation (APCM) coder is used for coefficients amplitudes. The number of bits is selected to assure a fixed quantization noise level for the amplitudes coefficients coding and varies beat-to-beat depending on the coefficients maximum amplitude.

\section{Data Packetization}

For the packetized communication purposes, an ECG data packet is generated each time a beat is coded thus enabling realtime transmission. There are two kinds of data that can be sent inside a packet: templates and residuals. The protocol data unit (PDU) used in both of them for transmission is illustrated in Fig. 5.

The PDU consists of the following fields.

- Template index: The index of the template used in template subtraction. This field is set to 0 when a new template is coded in the PDU and to 1 if the zero template is being used. Other numbers represent templates in the template database. The number of bits used in this field varies depending on the number of templates in the database. Decoder knows the maximum size at any time by counting the number of templates received.

- Number of leads: The number of coded ECG leads transmitted inside the data packet. 
TABLE I

Average Performance Results In CBR Mode For the Mit Dataset

\begin{tabular}{|c|c|c|c|}
\hline Target data rate $(b p s)$ & $\mathbf{2 5 0}$ & $\mathbf{5 5 0}$ & $\mathbf{8 5 0}$ \\
\hline Achieved data rate lead 1 & $248 \pm 25$ & $549 \pm 57$ & $849 \pm 90$ \\
\hline Achieved data rate lead 2 & $247 \pm 26$ & $550 \pm 56$ & $850 \pm 89$ \\
\hline Achieved RMS lead 1 & $25.3 \pm 7.4$ & $13.2 \pm 3.4$ & $9.3 \pm 2.4$ \\
\hline Achieved RMS lead 2 & $22.2 \pm 8.3$ & $11.7 \pm 3.1$ & $8.5 \pm 2.0$ \\
\cline { 2 - 4 } & \multicolumn{3}{|c|}{ (mean \pm sd) } \\
\cline { 2 - 4 }
\end{tabular}

- Number of coefficients for lead $j(j=1: n)$ : The number of coefficients selected to code lead $j$.

- Number of bits to code coefficients amplitudes in lead $j(j=1: n)$ : The number of bits used in the APCM coder to code the amplitudes for lead $j$.

- Coefficients amplitudes for lead $j(j=1: n)$ : The APCM coded amplitudes of the wavelet coefficients selected for lead $j$. The number of amplitudes is equal to the value written in the field Number of coefficients for lead $j$.

- First coefficient order for lead $j(j=1: n)$ : The first coefficient order coded with a PCM coder.

- Jumps for lead $j(j=1: n)$ : The coded jumps of the wavelet coefficients selected for lead $j$.

- RR interval: The number of samples between QRS complexes used to reconstruct the original beat duration.

\section{RESULTS AND DISCUSSION}

The MIT-BIH Arrhythmia database [18], which has been extensively used to evaluate ECG compression methods, has been used for performance evaluation. We have selected a subset of 11 two-leads records $(100,101,102,103,107,109,111,115$, $117,118,119)$ of 10 min duration. We will refer to them simply as the database. The sampling frequency is $360 \mathrm{~Hz}$ and the resolution is $11 \mathrm{bps}$. Average rate-distortion results on the data set were calculated for VBR and CBR modes. RMS $(\mu \mathrm{V})$ as well as transmission rate (bps) have been calculated for each beat.

\section{A. CBR Mode}

Simulation results have shown that in the application of the CBR mode to the MIT dataset, the method adjusts the transmission rate in a very accurate manner (see Table I). The standard deviation depends on the variability of RR interval because bit rate is adjusted every beat but in any case this deviation is small compared with the mean value. Note that target data rate cannot be exactly achieved due to steps of 1 bit are not possible since coefficients need more than one bit to be coded.

When a compression method is evaluated, a visual inspection of the resultant ECG data by a specialist to verify the clinical acceptability of the data is also very important. An excerpt of ECG signal containing an ectopic beat (record 109) and the reconstructed signal in CBR mode are shown in Fig. 6(a) and (c), respectively.

CBR mode can be very useful when the transmission rate has to fit to the available bandwidth in the communications network. Rate can be controlled in an accurate way guaranteing the desired transmission rate.

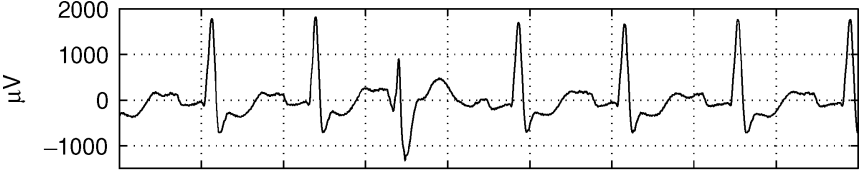

(a)

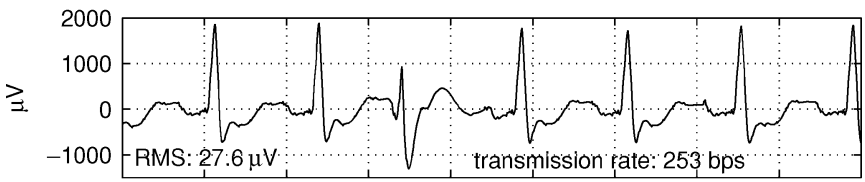

(b)

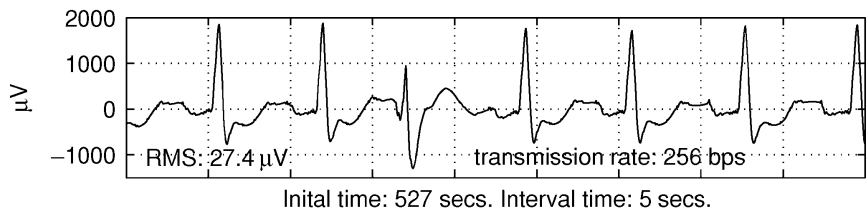

(c)

Fig. 6. Comparison between the original signal (record 109) and the reconstructed signal both in CBR and VBR with constant threshold modes. (a) Original signal (record 109). (b) Received signal. VBR mode with constant threshold (th $=30 \mu \mathrm{V})$, (c) Received signal. CBR mode (th $=255 \mathrm{bps}$ ).

TABLE II

Average Performance Results in VBR Mode for the Mit Data Set

\begin{tabular}{|c|c|c|c|}
\hline Target RMS $(\mu \mathrm{V})$ & $\mathbf{1 0}$ & $\mathbf{2 0}$ & $\mathbf{3 0}$ \\
\hline Achieved RMS lead1 & $9.8 \pm 0.7$ & $19.1 \pm 1.2$ & $27.5 \pm 2.3$ \\
\hline Achieved RMS lead2 & $9.6 \pm 0.4$ & $18.7 \pm 1.2$ & $26.7 \pm 2.6$ \\
\hline Achieved data rate lead1 & $703 \pm 191$ & $309 \pm 138$ & $198 \pm 115$ \\
\hline Achieved data rate lead2 & $623 \pm 174$ & $260 \pm 125$ & $160 \pm 104$ \\
\cline { 2 - 4 } & \multicolumn{3}{|c|}{ (mean \pm sd $)$} \\
\cline { 2 - 4 }
\end{tabular}

\section{B. VBR Mode}

1) VBR Mode With Constant Threshold: In VBR mode with constant threshold the results of the applied method not only adjust very well to the RMS mean distortion beat-to-beat but also present a very small RMS variance. These results can be seen in Table II. They have been obtained averaging the results from all the records in the database. The reconstructed signal from record 109 can be seen in Fig. 6(b).

When a high quality reconstructed signal is needed, the VBR mode with constant threshold can be used. The reconstruction error can be controlled with high precision permitting to achieve low reconstruction error in an accurate manner.

2) VBR Mode With Variable Threshold: In this mode, the threshold is adjusted according to the noise level measured beatto-beat in the preprocessing stage. Table III shows the results obtained when applying the VBR mode with variable threshold to the database. The first column shows the ECG record being tested. Columns 2 and 5, respectively, show the noise power measured in the first and second leads of the record, columns 3 and 6 show the reconstruction error obtained and columns 4 and 7 show, respectively, the transmission rate of the coded signal.

For a better understanding of the potential of this mode, the results in graphical format when the VBR mode with variable threshold is applied to record 101 lead 1 are shown in Fig. 7. The beat-to-beat measured noise in record 101 is illustrated in Fig. 7(a). Note that in this case, this value is used as threshold. 
TABLE III

VBR Mode With VARIABLE THRESHOLD PERFORMANCE FOR THE TESTED MIT DATA SET

\begin{tabular}{|c|c|c|c|c|c|c|}
\hline \multicolumn{7}{|c|}{ VBR with variable threshold } \\
\hline \multirow{3}{*}{ Record } & \multicolumn{7}{|c|}{ Compression results (mean \pm sd) } \\
\cline { 2 - 7 } & Noise & RMS & bps & Noise & RMS & bps \\
\hline $\mathbf{1 0 0}$ & $9.4 \pm 0.8$ & $9.1 \pm 0.8$ & $490 \pm 93$ & $9.1 \pm 0.9$ & $8.8 \pm 0.9$ & $513 \pm 85$ \\
\hline $\mathbf{1 0 1}$ & $13.3 \pm 10.9$ & $12.7 \pm 10.4$ & $558 \pm 136$ & $9.7 \pm 1.9$ & $9.4 \pm 1.9$ & $427 \pm 97$ \\
\hline $\mathbf{1 0 2}$ & $16.3 \pm 2.9$ & $16.7 \pm 3.2$ & $282 \pm 102$ & $19.8 \pm 7.6$ & $18.5 \pm 6.6$ & $397 \pm 106$ \\
\hline $\mathbf{1 0 3}$ & $11.1 \pm 0.6$ & $10.6 \pm 0.7$ & $377 \pm 74$ & $13.1 \pm 1.4$ & $12.4 \pm 1.4$ & $264 \pm 86$ \\
\hline $\mathbf{1 0 7}$ & $36.1 \pm 4.3$ & $34.4 \pm 4.6$ & $375 \pm 89$ & $30.0 \pm 2.9$ & $28.0 \pm 2.9$ & $338 \pm 78$ \\
\hline $\mathbf{1 0 9}$ & $15.6 \pm 4.2$ & $14.4 \pm 4.0$ & $440 \pm 111$ & $10.5 \pm 7.6$ & $10.0 \pm 7.0$ & $556 \pm 122$ \\
\hline $\mathbf{1 1 1}$ & $17.5 \pm 4.0$ & $16.4 \pm 3.6$ & $316 \pm 108$ & $13.8 \pm 5.2$ & $13.2 \pm 4.7$ & $480 \pm 131$ \\
\hline $\mathbf{1 1 5}$ & $17.7 \pm 1.9$ & $16.8 \pm 1.9$ & $218 \pm 73$ & $9.9 \pm 0.2$ & $9.5 \pm 0.4$ & $342 \pm 62$ \\
\hline $\mathbf{1 1 7}$ & $15.2 \pm 1.1$ & $14.8 \pm 1.2$ & $213 \pm 64$ & $16.2 \pm 1.3$ & $15.8 \pm 1.4$ & $243 \pm 70$ \\
\hline $\mathbf{1 1 8}$ & $26.0 \pm 11.5$ & $24.4 \pm 11.2$ & $535 \pm 137$ & $24.9 \pm 11.1$ & $23.3 \pm 10.6$ & $535 \pm 128$ \\
\hline $\mathbf{1 1 9}$ & $20.1 \pm 7.0$ & $19.7 \pm 7.0$ & $375 \pm 163$ & $14.8 \pm 0.8$ & $14.8 \pm 1.3$ & $402 \pm 176$ \\
\hline
\end{tabular}

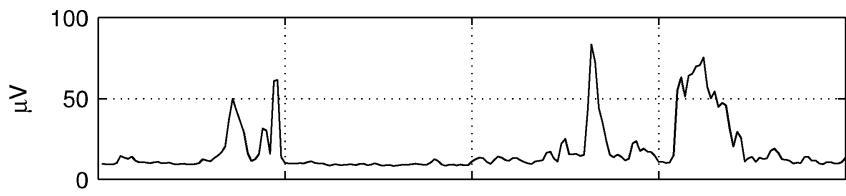

(a)

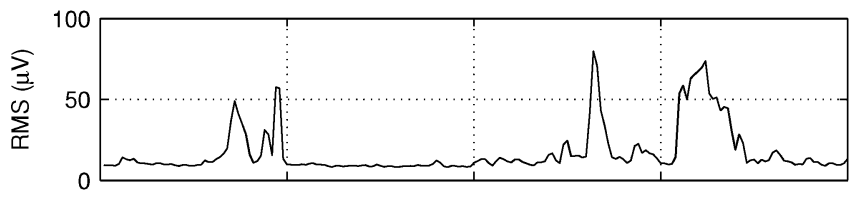

(b)

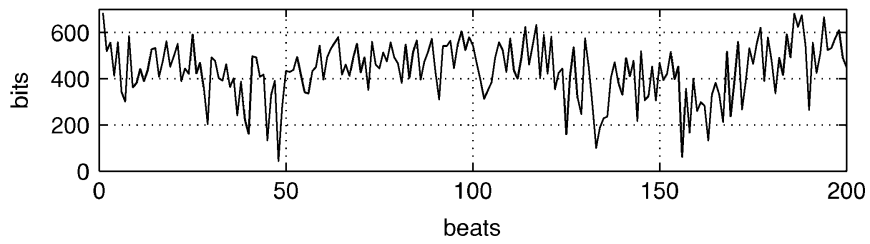

(c)

Fig. 7. VBR mode with variable threshold for record 101. (a) Measured noise. (b) Reconstruction error (VBR mode with variable threshold). (c) Bits per beat.

Fig. 7(b) shows the reconstruction error between the original record (with noise) and the reconstructed signal. As it can be seen, it fits very well the threshold showing the accuracy of the VBR mode. Finally, the number of bits needed to transmit each beat is presented in Fig. 7(c). It is also worthy a visual check of the reconstruction error. Fig. 8(a) shows five original beats from record 101. The received signal in VBR mode with constant and variable threshold are shown in Fig. 8(b) and (c), respectively. Values of reconstruction error as well as the number of bits needed to code each beat are given in the figure.

VBR mode with variable threshold can be very useful indeed. One reason is that by removing white noise beat-to-beat from the ECG signal, the bits used for coding are dedicated only to signal coefficients thus cleaning the signal at the same time that compression is achieved. In a normal clinical practice, the noise introduced in the acquisition of the ECG is not usually below $10 \mu \mathrm{V}$, leading this value to a compression factor high enough to be useful for compression purposes (see Table III, for an example in MIT-BIH database). Another important reason for using the VBR mode with variable threshold is that it prevents the transmission rate from increasing dramatically when a beat is contaminated with high power noise. This effect can

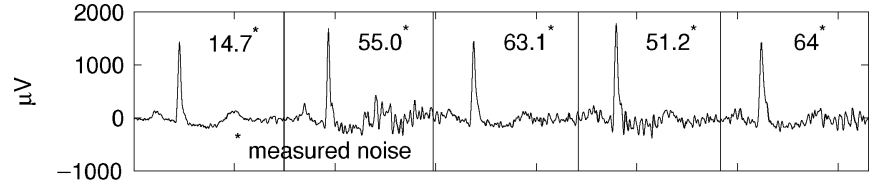

(a)

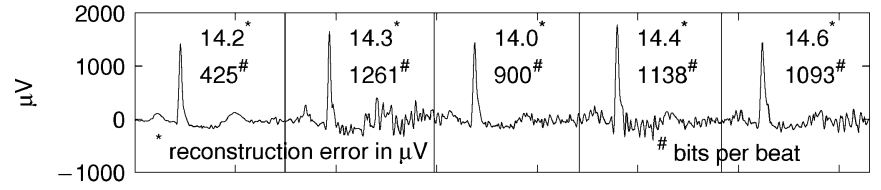

(b)

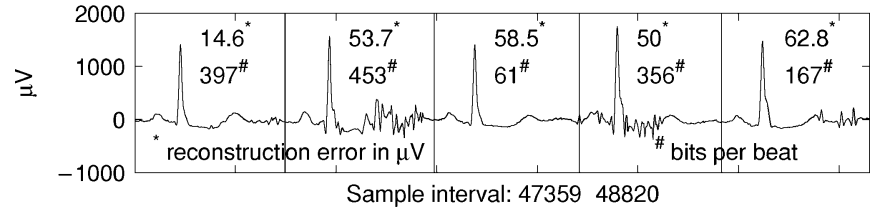

(c)

Fig. 8. Comparison between original signal (record 101) and reconstructed signals in VBR mode. (a) Original signal (record 101). (b) Received signal in VBR with constant threshold (th $=15 \mu \mathrm{V}$ ). (c) Received signal in VBR with variable threshold.

be appreciated in Fig. 8. Beats from 2 to 4 are contaminated with a high power noise. Working in VBR mode with constant threshold [see Fig. 8(b)], the bit rate increases dramatically in order to maintain the reconstruction error fixed by the threshold. If the operation mode is VBR with variable threshold, the increase in the noise will lead to an increase in the threshold, thus preventing this dramatic increase in the transmission rate [see Fig. 8(c)]. Using VBR with variable threshold we can avoid to spend bits in noise coding. Also note that in this mode, the user do not have to care about setting the compression threshold manually to obtain a good quality reconstructed signal suitable for diagnosis.

\section{Clinical Evaluation}

Strategy VBR with variable threshold has been clinically evaluated using the database. We have selected only this strategy because it represents a novelty from other compression methodologies. The approach adopted in the clinical evaluation was the proposed in [19]. In this paper, a weighted mean opinion score (WMOS) error was defined as the average of two MOS error tests. The first one is a blind test which analyzes both the original and reconstructed signals individually (each one is shown in a different sheet of paper). Interpretation of different ECG signal parameters ( $\mathrm{P}$ and T waves, QRS complex, ST level and abnormal beats) is requested to the cardiologist. The second one is a semi-blind test which compares the original and reconstructed signals together (both are in the same sheet of paper). In this test, the cardiologist provides an evaluation of the similarity between original and reconstructed signals. In our implementation, $10 \mathrm{~s}$ of both leads were considered for evaluation. Thus, the VBR mode with variable threshold was applied to the database (both leads) and the recostructed signals (as well as the original) were evaluated following the described procedure by 
TABLE IV

Clinical EVALUATION OF VBR MODE With VARIABLE THRESHOLD USING WMOS error

\begin{tabular}{|c|c|c|c|c|c|c|c|c|c|c|c|}
\hline Record & $\mathbf{1 0 0}$ & $\mathbf{1 0 1}$ & $\mathbf{1 0 2}$ & $\mathbf{1 0 3}$ & $\mathbf{1 0 7}$ & $\mathbf{1 0 9}$ & $\mathbf{1 1 1}$ & $\mathbf{1 1 5}$ & $\mathbf{1 1 7}$ & $\mathbf{1 1 8}$ & $\mathbf{1 1 9}$ \\
\hline Initial sample & 1001 & 47180 & 1001 & 1001 & 93200 & 27298 & 91606 & 1001 & 1001 & 202082 & 202082 \\
\hline WMOS $_{\text {error }}$ & 2.5 & 5.0 & 10.0 & 0.0 & 15.6 & 0.0 & 5.0 & 5.0 & 0.0 & 7.5 & 5.0 \\
\hline \multicolumn{10}{|c|}{ WMOS } \\
\hline
\end{tabular}

TABLE V

MaXimum EXecution Times for THE CODING AND DeCOding Process IN DIFFERENT PCs

\begin{tabular}{|c|c|c|}
\hline PC characterisics & Coding & Decoding \\
\hline Centrino 1.4 Ghz 512 MB RAM & $20 \mathrm{~ms}$. & $3 \mathrm{~ms}$. \\
\hline Pentium II 300 MHz 256 MB RAM & $90 \mathrm{~ms}$. & $16 \mathrm{~ms}$. \\
\hline
\end{tabular}

two cardiologists from different hospitals. Table IV shows these results. The first row presents the name of the evaluated records. The second one shows the initial sample of the interval being evaluated. Final sample is the initial sample plus 3600 (10 s). The WMOS error value can be seen in the third row. Finally, the forth row shows the clinical interpretation of the WMOS error value as shown in Table $\mathrm{V}$ of [19]. Both leads of the records are evaluated jointly because cardiologists point out that both of them are needed to give a diagnosis. The lower the $\mathrm{WMOS}_{\text {error, }}$ the better the quality of the reconstructed signal. Attending to the clinical evaluation, the quality of the reconstructed signals was very good in all the records except 107 where the quality was good, thus validating the method for clinical use.

\section{Real-Time Performance}

Usually, the maximum number of leads an ECG signal can present is 8 (extra leads in a 12-lead ECG record are calculated from the original 8). Therefore, we have used 8leads ECG records to evaluate the execution times of the coding and decoding processes. Since we have been working with the MIT-BIH database, we have created 8-lead records from those of the database replicating both leads four times. Each record of the database was coded/decoded separately as it would be done in a real-time operation. CBR mode with a high threshold (th $=1200$ bps) was used because its thresholding process is more time demanding than VBR mode. Average execution times were obtained for the database and the maximum times among them are reported in Table V. Results are given in milliseconds required to code 1 second of original signal.

Results of Table V clearly show that even for a PC with a Pentium II $300 \mathrm{MHz}$ processor with $256 \mathrm{MB}$ of RAM there is no problem for achieving real-time functionality. The programming language used for the coder was $\mathrm{C}$ because of its efficiency.

\section{CONCLUSION}

This paper presents a new wavelet-based ECG packetized compression method with two operational modes, CBR and VBR. The method is particularly suitable for real-time telecardiology applications. In CBR mode, the output bite rate is approximately constant. This mode is useful when dealing with networks whose available bandwidth is small and well defined, such as $2.5 \mathrm{G}$ wireless networks. In VBR mode, the output data rate varies but the reconstructed error is bounded. VBR can be performed in two operational modalities. VBR mode with variable threshold adjusts automatically beat-to-beat the threshold according to the measured noise in the beat. VBR with constant threshold maintains the threshold value through a time interval and it is set up by the user. The compression method has been tested on records from MIT-BIH Arrhythmia database, showing an accurate behavior when working in both modes. VBR mode with variable threshold has been clinicaly evaluated in the WMOS error scale obtaining results classified as good and very good.

\section{ACKNOWLEDGMENT}

The authors would like to thank Dr. L. Ramos and Dr. P. Serrano for their support in the clinical evaluation of the method.

\section{REFERENCES}

[1] G. Molinari, G. Reboa, M. Frascioa, M. Leoncini, A. Rolandi, C. Balzan, and A. Barsotti, "The role of telecardiology in supporting the decisionmaking process of general practitioners during the management of patients with suspected cardiac events,"J. Telemed. Telecare, vol. 8, no. 2, pp. 97 101, 2002.

[2] R. S. H. Istepanian and A. A. Petrosian, "Optimal zonal wavelet-based ECG data compression for a mobile telecardiology system," IEEE Trans. Inf. Technol. Biomed. vol. 4, no. 3, pp. 200-211, Sep. 2000.

[3] R. Fogliardi, E. Frumento, M. A. V. D. Rincón, and M. Fregonara, "Telecardiology: Results and perspectives of an operative experience," J. Telemed. Telecare, vol. 6, no. Sup. 1, pp. 162-164, 2000.

[4] B. Woodward, R. Istepanian, and C. Richards, "Design of a telemedicine system using a mobile telephone," IEEE Trans. Inf. Technol. Biomed., vol. 5, no. 1, pp. 13-15, Mar. 2001.

[5] M. L. Sedgewick, K. Dalziel, J. Watson, D. J. Carrington, and S. M. Cobbe, "Performance of an established system of first responder out-of-hospital defibrillation. The results of the second year of the heartstart scotland project in the utslein style," Resucitation, vol. 26, pp. 75-78, 1993.

[6] S. Pavlopoulos, E. Kyriacou, A. Berler, S. Dembeyiotis, and D. Koutsoris, "A novel emergency telemedicine system based on wireless comunication technology-AMBULANCE," IEEE Trans. Inf. Technol. Biomed., vol. 2, no. 4, pp. 261-267, Dec. 1998.

[7] K. Shimizu, "Telemedicine by mobile communication," IEEE Eng. Med. Biol. Mag., pp. 32-44, Jul./Aug. 1999.

[8] M. Metcalfe, "The next-generation internet," IEEE Internet Comput., vol. 4, pp. 58-59, Jan./Feb. 2000.

[9] A. Jamalipour and P. Lorenz, "Merging IP and wireless networks [guest editorial]," IEEE Wireless Commun., vol. 10, no. 5, pp. 6-7, Apr. 2003.

[10] M. L. Hilton, "Wavelet and wavelet packet compression of electrocardiograms," IEEE Trans. Biomed. Eng., vol. 44, no. 5, pp. 394-402, May 1997.

[11] Z. Lu, D. Y. Kim, and W. A. Pearlman, "Wavelet compression of ECG signals by the set partitioning in hierarchical trees algorithm," IEEE Trans. Biomed. Eng., vol. 47, no. 7, pp. 849-856, Jul. 2000.

[12] S. G. Miaou, H. L. Yen, and C. L. Linn, "Wavelet-based ECG compres sion using dynamic vector quantization with tree codevectors in single codebook," IEEE Trans. Biomed. Eng., vol. 47, pp. 849-856, Jul. 2002.

[13] A. I. Hernández, F. Mora, G. Vilegas, and G. P. G. Carrault, "Real-time ECG transmission via internet for nonclinical applications," IEEE Trans. Inf. Technol. Biomed., vol. 5, no. 3, pp. 253-257, Sep. 2001.

[14] J. P. Martínez, R. Almeida, S. Olmos, A. P. Rocha, and P. Laguna, "A wavelet-based ECG delineator: Evaluation on standard databases," IEEE Trans. Biomed. Eng., vol. 51, no. 4, pp. 570-581, Apr. 2004.

[15] J. García, L. Sörnmo, S. Olmos, and P. Laguna, "Automatic detection of ST-T complex changes on the ECG using filtered RMS difference series: Application to ambulatory ischemia monitoring," IEEE Trans. Biomed. Eng., vol. 47, no. 9, pp. 1195-1201, Sep. 2000.

[16] B. Bradie, "Wavelet packet-based compression of single lead ECG," IEEE Trans. Biomed. Eng., vol. 43, no. 5, pp. 493-501, May 1996. 
[17] A. Alesanco, S. Olmos, J. Salcedo, R. Istepanian, and J. García, "A realtime multilead ECG compression and de-noising method for a mobile telecardiology system," Int. Congr. Comput. Bioeng., vol. 1, pp. 237-242, Sep. 2003.

[18] MIT-BIH Arrhythmia Database CD-ROM. Harvard-MIT Division of Healh Sciences and Technology, May 1997.

[19] Y. Zigel, A. Cohen, and A. Katz, "The weighted diagnostic distortion (WDD) measure for ECG signal compression," IEEE Trans. Biomed. Eng., vol. 47, no. 11, pp. 1422-1430, Nov. 2000.

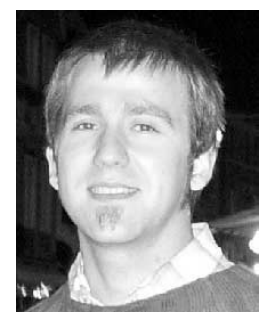

Álvaro Alesanco was born in Ezcaray, Spain, in 1977. He received the Master's degree in telecommunications engineering from the University of Zaragoza (UZ), Zaragoza, Spain, in 2001.

Currently, he is an Assistant Professor of Communication Networks in the Telematics Engineering area in UZ. He is also Visiting Researcher at Mobile Information and Network Technologies Research Center, Kingston University, Kingston upon Thames, London, U.K. He has undergone different research stages working on telemedicine in Australia and the United Kingdom. His research interests are ECG and echocardiography video coding and transmission in wireless e-health environments.

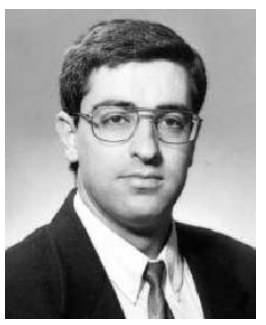

Salvador Olmos (A'01-M'03) was born in Valencia, Spain, in 1969. He received the Industrial Engineering degree and the Doctor degree (Ph.D.) from the Polytechnic University of Catalonia, Spain, in 1993 and 1998, respectively.

Currently, he is an Associate Professor of Signal Processing and Communications in the Department of Electronics and Engineering and Communications at the University of Zaragoza, Spain. From August 2000 to August 2001, he was with the Department of Electroscience, Lund University, Sweden, supported by a postdoctoral research grant from Spanish Government. His professional research interests are in signal processing of biomedical signals as well as medical imaging.

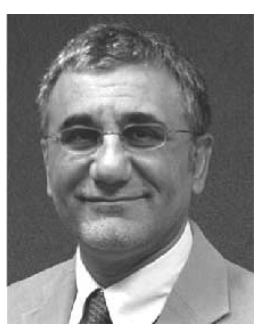

Robert S. H. Istepanian (SM'00) received the Ph.D. degree from the Electronic and Electrical Engineering Department Loughborough University, U.K., in 1994.

From 1984 to 1998 , he worked in different overseas industrial and academic positions. In 1988, he was a Visiting Research Fellow in the Department of Electronic and Electrical Engineering, Loughborough University, U.K. He was with the same department from 1994 to 1995 as a Postdoctoral Research Fellow. From 1996 to 1999, he was a Senior Lecturer at the University of Portsmouth, U.K. Between 1999-2000, he was an Associate Professor at the Universities of Western Ontario and Ryerson in Toronto, ON, Canada. From 2000 to 2003, he was a Senior Lecturer and head of the (Mobile Information Engineering and E-Med Systems) research group in the Department of Electronic and Computer Engineering Brunel University- West London. In 2003, he joined the School of Computing and Information Systems, Kingston University, London, as Professor of Data Communications and Director of Mobile Information and Network Technologies (MINT) Research Centre in the University. Since 1996, he has been involved in research and management of research projects from U.K. and EU. He is recipient, investigator, and coinvestigator of research grants from EU, EPSRC and other research visiting grants from the British Council and Royal Society on intelligent computational systems applied to telesurgery systems. He has published more than 140-refereed journal and conference papers and three books including chapters in these books in the areas of biomedical signals processing and mobile communications for healthcare and $\mathrm{m}$-health technologies.

Prof. Istepanian is a Fellow of the Institute of Electrical Engineers. He currently serves on the editorial boards of the IEEE TRANSACTIONS ON INFORMATION TECHNOLOGY IN BIOMEDICINE and was one of the founding special area editors of the IEEE TRANSACTIONS ON INFORMATION TECHNOLOGY IN BIOMEDICINE and telemedicine and e-health since 1997. He has served as guest editor of three special issues of the IEEE TRANSACTIONS ON INFORMATION TECHNOLOGY IN BIOMEDICINE and the IEEE TRANSACTION ON NANOBIOSCIENCE since 1999. He is also an Associate Editor of the Journal of Mobile Multimedia and Journal on Information Technology in Healthcare.

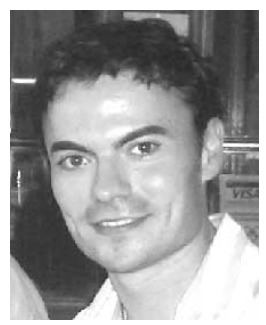

José García was born in Zaragoza, Spain, in 1971. He received the M.S. degree in physics and the Ph.D. degree (with Honors) from the University of Zaragoza (UZ), Zaragoza, Spain, in 1994 and 1998, respectively.

He is with the Department of Electronics Engineering and Communications in the Polytechnic Center of UZ. Currently, he is an Associate Professor in the Telematics Engineering area and member of the Aragón Institute of Engineering Research (I3A). He is the founder and responsible of the Telemedicine Group in the I3A. He is recipient, investigator, and coinvestigator of research grants from the Ministry of Science and Technology and the Sanitary Research Funds in the area of telemedicine applications and networks. His work is also supported by major industrial and mobile companies such Telefónica Móviles in the area of wireless communications for health. He has undergone different research stages in the United States, Sweden, and Austria. He has published more than 40 refereed international journal and conference papers and mostly in the areas of wireless telemedicine and biomedical signal processing. He is also reviewer of several journals on the topic. His research interests are in telemedicine, biomedical signal processing for transmission, wireless communications, and other related topics. 